\title{
Benign Uterine Corpus Mixed Epithelial and Mesenchymal Neoplasm
}

National Cancer Institute

\section{Source}

National Cancer Institute. Benign Uterine Corpus Mixed Epithelial and Mesenchymal

Neoplasm. NCl Thesaurus. Code C6335.

A non-metastasizing neoplasm that arises from the uterine corpus and is composed of epithelial and mesenchymal elements. Representative examples include adenomyoma and adenofibroma. 